\title{
No diabetes-associated mutations in the coding region of the hepatocyte nuclear factor-4 $\gamma$ gene (HNF4G) in Japanese patients with MODY
}

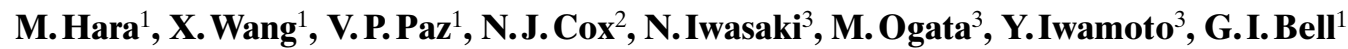 \\ ${ }^{1}$ Howard Hughes Medical Institute, The University of Chicago, Chicago, Illinois, USA \\ ${ }^{2}$ Departments of Human Genetics and Medicine, The University of Chicago, Chicago, Illinois, USA \\ ${ }^{3}$ Diabetes Centre, Tokyo Women's Medical University, Tokyo, Japan
}

\begin{abstract}
Aims/hypothesis. Mutations in the transcription factor hepatocyte nuclear factor (HNF)- $4 \alpha$ are the cause of one form of maturity-onset diabetes of the young, MODY1. The HNF- $4 \gamma$ is structurally related to HNF- $4 \alpha$ and is expressed together with HNF- $4 \alpha$ in pancreatic islets. We therefore tested the hypothesis that genetic variation in the HNF-4 $\gamma$ gene $(H N F 4 G)$ is associated with MODY in Japanese subjects.

Methods. We screened the protein coding region of HNF4G (exons 3-11) for mutations in 57 unrelated Japanese subjects with MODY by amplifying each exon and adjacent intron region using the polymerase chain reaction (PCR) and specific primers and then directly sequencing the PCR products. The frequency of each variant was compared between patients with MODY and a group of non-diabetic subjects.
\end{abstract}

Results. We found ten sequence variants, two of these were located in exons: exon 6 , a silent substitution in codon 144, c.432A/G and exon 7, a G-to-A substitution in codon 190 (c.570G/A) resulting in a conservative Met-to-Ile substitution (M/I190) in the putative ligand-binding region of HNF- $4 \gamma$ protein. The remaining eight variants were located in introns. There was no significant difference in the frequency of these polymorphisms between subjects with MODY and non-diabetic control subjects.

Conclusion/interpretation. Genetic variation in the coding region of $H N F 4 G$ is unlikely to be a major cause of MODY in Japanese people. [Diabetologia (2000) 43: 1064-1069]

Keywords Maturity-onset diabetes of the young, MODY, transcription factor, nuclear receptor, HNF$4 \gamma$, diabetes mellitus, insulin, genetics, mutation.
The discovery that mutations in transcription factors expressed in the pancreatic beta cell can cause diabetes mellitus [1] has led many groups to search for mutations in islet transcription factors in patients who appear to have a monogenic form of this disorder including maturity-onset diabetes of the young (MODY) and early-onset autosomal-dominant Type II (non-insulin-dependent) diabetes mellitus. Diabe-

Received: 3 March 2000 and in final revised form: 11 May 2000

Corresponding author: M. Hara, DDS, PhD, Howard Hughes Medical Institute, The University of Chicago, 5841 S. Maryland Ave., MC1028, Chicago, IL 60637, USA

Abbreviations: HNF: Hepatocyte nuclear factor, IPF: insulin promoter factor, MODY: maturity-onset diabetes of the young. tes-associated mutations have been found in a nuclear receptor (hepatocyte nuclear factor (HNF)- $4 \alpha$ [2]), homeodomain-containing proteins (HNF-1 $\alpha$ [1], and HNF-1 $\beta$ [3], insulin promoter factor (IPF)-1 [4] and islet-1 [5]) and a basic helix-loop-helix (bHLH) protein (NeuroD1/BETA2) [6]. In addition, mutations in the glycolytic enzyme glucokinase can cause MODY [7]. The molecular mechanism(s) by which mutations in the transcription factors described above cause diabetes are poorly understood but could include effects on pathways regulating insulin secretion as well as islet development. Mutations in the HNF-1 $\alpha$ and glucokinase genes are the most common causes of monogenic forms of diabetes amongst Europeans [8]. They seem, however, to be less important in Asians (Japanese and Chinese) and the major gene(s) responsible for MODY in Asians 
Table 1. Sequences of primers for amplification and sequencing of the coding region of $H N F 4 G$

\begin{tabular}{|c|c|c|c|}
\hline Region & Forward primer ( $\left.5^{\prime}-3^{\prime}\right)$ & Reverse primer (5'-3') & $\begin{array}{l}\text { Product size } \\
\text { (base pairs) }\end{array}$ \\
\hline Exon 3 & GCCAATGAATGGAGTGTTATGAG & CTGTGTACATACCCACACGTTC ${ }^{\mathrm{a}}$ & 427 \\
\hline Exon 4 & GCTGATTGTTCCTATACCTTC ${ }^{a}$ & GATGTCCTTGGGCTAAATTC & 389 \\
\hline Exon 5 & GAGCTCTAGTGACAAACCTG ${ }^{\mathrm{a}}$ & GCATGATGCCTACATGGAC & 360 \\
\hline Exon 6 & CAATAGTGCCTACTTCTATGGC ${ }^{a}$ & CAGTTGTGCCTCTTGATAG & 444 \\
\hline Exon 7 & GGCTTAATGATGGCATGTATC ${ }^{a}$ & GCCAGTGTCTGTAAATACTTG & 338 \\
\hline Exon $8^{\mathrm{b}}$ & CTATTCCTTCAAGGGTAGAC ${ }^{\mathrm{a}}$ & GTCTCTCTGATGGGTCTTGC & 832 \\
\hline Exon 9 & GTCTCACACTGTAATTAGG ${ }^{\mathrm{a}}$ & & \\
\hline Exon 10 & GCCAATGTACAGTGAAAGCTG ${ }^{\mathrm{a}}$ & СAGTCTTCTACTGCTGAGTC & 454 \\
\hline Exon 11 & CTAACAGTACGTCATGGGCC ${ }^{\mathrm{a}}$ & CTCAGATATACATTGGTTGC ${ }^{a}$ & 499 \\
\hline
\end{tabular}

${ }^{a}$ Denotes primers used for sequencing the PCR product

${ }^{\mathrm{b}}$ Because intron 8 was only 130 bp, exons 8 and 9 were amplified together and the PCR product was sequenced using two forward primers

Table 2. Polymorphisms in $H N F 4 G$ in Japanese subjects

\begin{tabular}{|c|c|c|c|c|c|c|c|}
\hline \multirow[t]{2}{*}{ Location } & \multirow[t]{2}{*}{ Nucleotide $^{a}$} & \multirow{2}{*}{$\begin{array}{l}\text { Nucleotide } \\
\text { change }\end{array}$} & \multirow[t]{2}{*}{ Designation } & \multirow[t]{2}{*}{ Amino acid change } & \multirow[t]{2}{*}{ Designation } & \multicolumn{2}{|c|}{ Frequency of major allele } \\
\hline & & & & & & MODY & Non-diabetic \\
\hline $\begin{array}{l}\text { Exon } 6 \\
\quad \text { Codon } 144\end{array}$ & 432 & $\mathrm{~A} / \mathrm{G}$ & c. $432 \mathrm{~A} / \mathrm{G}$ & $\mathrm{K}(\mathrm{AAA})>\mathrm{K}(\mathrm{AAG})$ & & A- -0.93 & 0.87 \\
\hline Intron 6 & nt-78 & $\mathrm{C} / \mathrm{G}$ & IVS6nt-78C/G & & & C- 0.47 & 0.51 \\
\hline $\begin{array}{l}\text { Exon } 7 \\
\quad \text { Codon } 190\end{array}$ & 570 & $\mathrm{G} / \mathrm{A}$ & c.570G/A & M (ATG) > I (ATA) & M/I190 & G-0.46 & 0.54 \\
\hline Intron 7 & $\mathrm{nt}+6$ & $\mathrm{~T}_{10-12} \mathrm{~A}_{2-3}$ & & & & & \\
\hline Intron 9 & $\begin{array}{l}\text { nt-94 } \\
\text { nt-19 }\end{array}$ & $\begin{array}{l}\mathrm{A} / \mathrm{G} \\
\mathrm{A} / \mathrm{T}\end{array}$ & $\begin{array}{l}\text { IVS9nt-94A/G } \\
\text { IVS9nt-19A/T }\end{array}$ & & & $\begin{array}{l}\text { A- }-0.54 \\
\text { A- } 0.58\end{array}$ & $\begin{array}{l}0.52 \\
0.60\end{array}$ \\
\hline Intron 10 & $\mathrm{nt}+34$ & $\mathrm{~T} / \mathrm{A}$ & IVS10nt+34T/A & & & $\mathrm{T}-0.93$ & 0.89 \\
\hline 3'-UTR & $\begin{array}{l}1289 \\
1350\end{array}$ & $\begin{array}{l}\text { G/A } \\
\text { G/A }\end{array}$ & $\begin{array}{l}\text { c. } 1289 \mathrm{G} / \mathrm{A} \\
\text { c. } 1350 \mathrm{G} / \mathrm{A}\end{array}$ & & & $\begin{array}{l}\text { G- } 0.56 \\
\text { G-0.56 }\end{array}$ & $\begin{array}{l}0.64 \\
0.64\end{array}$ \\
\hline
\end{tabular}

${ }^{a}$ Nucleotide (nt) numbering - the A of the ATG of the initiator Met codon is denoted nucleotide +1 and the lower case $\mathrm{c}$ for cDNA in front of the nucleotide number indicates that the reference sequence is the cDNA sequence (if the reference sequence was the genomic sequence, lower case $g$ for genomic would precede the nucleotide number). The nucleotide location within an intron is numbered relative to the splice donor (+) or acceptor (-) site. The frequency of each substitution was determined in 57 unrelated MODY patients and 50 unrelated non-diabetic (by oral glucose tolerance testing) subjects. The polymorphism located in intron $7, \mathrm{nt}+6$ is a complex polymorphism with variation in length of both the T-tracts and

is not known. The HNF- $4 \gamma$ is structurally-related to HNF- $4 \alpha$ and like HNF- $4 \alpha$ is also expressed in islets [9]. Thus, it is a plausible candidate diabetes gene. Genetic variation in the HNF-4 $\gamma$ gene $(H N F 4 G)$ was not the cause of diabetes in 32 multigenerational families of European ancestry with early-onset autosomal-dominant Type II diabetes unlinked to known MODY genes [10]. We examined the relation between genetic variation in $H N F 4 G$ and MODY in Japanese.
A-tracts and $76 \%$ and $72 \%$ of the MODY patients and control subjects were heterozygous at this site. In addition to the polymorphisms described above, we also noted another polymorphism in intron 6 (IVS6nt+90G/T) that we did fully characterise in these samples. There is statistically significant linkage disequilibrium among the polymorphisms listed above with near perfect linkage disequilibrium between the following pairs: c.432A/G and IVS6nt-78C/G; M/I190 and IVS9nt94 A/G; and c.1289G/A and c.1350G/A. From the polymorphisms found in Japanese five (IVS6nt-78C/G, IVS9nt-94A/G, IVS9nt-19A/T, IVS10nt+34T/A and c.1350G/A) were not reported in [10]. UTR-untranslated region

\section{Subjects and methods}

Subjects. The study cohort consisted of 57 unrelated Japanese subjects with a diagnosis of MODY, the clinical features of which have been described [11]. These subjects have previously been screened for mutations in the HNF- $1 \alpha,-1 \beta$ and $-4 \alpha$, IPF-1, NeuroD1/BETA2, Nkx2.2, HNF-3 $\beta$ and DCoH genes. Mutations in the HNF-1 $\alpha$ gene have been excluded as the cause of MODY in each of these subjects. This group does include one subject with a nonsense mutation in the HNF-1 $\beta$ gene and two subjects with putative diabetes-associated mutations in the HNF- $4 \alpha$ gene. The control group consisted of 50 unrelated non-diabetic (by oral glucose tolerance testing) subjects. All subjects were recruited from Tokyo Women's Medical University. This study was approved by the Institutional Review Board of Tokyo Women's Medical University and 
GGCCGGGCTTTGGGACTTGCTGGCCCGACCGCCAGTCGCCCTGGGGCCTTGGGAACAGGGAAATGCGCGCNCCGAGNCGGTGNCGGCCTCGCTGCTTTCAGTTTTCATTGAGTCTGCTGGT TACTGTTTAACGTCCCCACCCACGTTTCAGTTTGTTTAGTAAATCGATCGGACTGTGAGAGTCAGGCCGCTGCCCTGTCGCCATTGAGCAAAGCTAATATTCTCCCAGCATATGGTCTAAA ATCTTTGCTAGAGATCGGGAAAAAGAAACAAACAAACAAACAAACAACAACAAAATCCTTAAGCTTCCTCTCCCAAGTCCCCCCATCCCCACCCCCACTTCCCACCTCCTCGCTTTCAGCA AACTTCCCAGCCTGGGAAAAGTGCGGCGCGGGACAGCGCGGCGGGGTGGGTGGGGGACCTGCTGCCGGGAGATGCGGCGGCGGCCGCGCGGCTGACTGCGACTCGGGAGCTCCGGGAGCGG

$$
\text { * Exon } 1
$$

CCCGCGCAGGTAAGTTTCTCCGCCGCCGCCCGGGGNCCGGGCTGGCCGCAGCGCACCGCCGGCGGCTGCCGGGCAGGA GGAGCCTCGGTTCCCCTCGGGCGGCTGAAGGAGGGGCTGAGCC TCAGGACCCCTAGGCCAGGCCGTGTTGAAAGCAGATACCCCGAGGCCTCGCCCAGCCTGCGCTGGAGGGAAGAAGGCGCTGGAG GTGCGGCAGCGGGCAGTGGGGGGACCGGGGAATTGAA

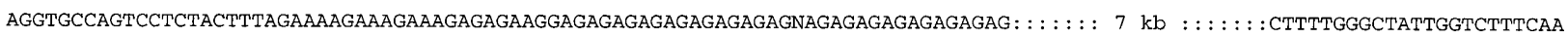
Exon 2

CATGCAGTTAGGGATAAAAGTAAATCATTTAATCTATGCAGCATGTCACTCAATAGTATACTTTTGGAAATTGTTTCCACAG GAGCACCAGCGAAagCAGCCAGTCTGAGATATTGACAC TACAGAAAAAATGACAGCTTACTCCTTGTATTGATTCTACTCTTCTCTACAAATATAGACTCCGTTCCCTACCACAGCCTT GTAAGTGGTGCCCGCTGAGGCTGCTAAATTGCTTTGTAA ATTATTTATTACTTGGACACCTTGTAAAGACCTCTTTNCTTCTTGTCTGACTTGCTTGTGCTTTCGTATATACTAAAAGGTCTCAAAGTGTACACTGACCACAACCTCCCTTATGAAATCC TTT: : : : : : $1 \mathrm{~kb}:::::::$ CCTGGTGAAGAAGGTTACAGTTTTACAGCCCCTCCCATTGCAGCTCTTAAGTCACTCAGTTACAGTTAACTTTGGATTAGCACTCACAGATTGAAAGC Exon $2 \mathrm{~A}$ M $\quad D \quad M \quad A \quad N \quad Y \quad S$ AAAACACATCAAAACACTCATCACGCACTCTGGGCTTGTGGTGCCACTTGTATGTGTGTTTCTAAATCAATGATGAGGGTATCAg AACCAATACTGGACATGGACATGGCAAATTACAGTG $\begin{array}{lllllllllllllllllllllll}E & V & L & D & P & T & Y & T & T & \text { L } & \text { E } & \text { F } & \text { E } & \text { T } & \text { M } & Q & \text { I } & \text { L } & Y & \text { N } & S & S\end{array}$ AAGTTTTGGACCCAACTTACACAACTTTGGAGTTTGAAACTATGCAGATTCTATATAATTCAAGTG GTGAGTTATCATCTGTTTATAGATGTAAAGAAAAGTAAGTATAATTGGAGAAGGT GGAAGACTGAGCTTCCAAAGTTGTGGCTCAATGTTTTTTTTGCTGGAGAATTTAGGGCTTGCTTTTAAGG:CACAGATCTGAGCTTTTGCACTTCATTAC: : : : : : : 5 kb : : : : : : CGGGCTGGTATTTATTGTCATGGAACTGATAAACTTCCCTTGGGAAAGAGCATAGTTGGAGAACTAAGCCCTATGGTGTCCCAAAGTTAAATGTTCAGAAATAAGAAAGGAGAGCTTAGAA CGGAAGCAGCCAGCCAGGGTTAAAAGGAAAGCACCAGTGTGGGGTCTCCAAGAAGCCAATGAATGGAGTGTTATGAGCCTATAAATAATTAGTAGGACAATTTAGTCAGGAAATAGTACTA Exon 3 (1 $D$ S $S$ A $P$ E $T$ T Met Asn Thr Thr Asp Asn Gly Val Asn Cys teu Cys Ala Ile Cys Gly ACTCTAACTGTAATCTTCCTTTTTTATTGACAG ATAGTTCTGCCCCAGAGACAAGT ATG AAT ACC ACA GAC AAC GGT GTC AAC TGT CTG TGT GCT ATC TGT GGG

Asp Arg Ala Thr Gly Lys His Tyr Gly Ala Ser Thr Cys Asp Gly Cys Lys Gly Phe Phe Arg Arg Ser Ile Arg Lys Ser His Ile Tyr GAC AGA GCA ACA GGA AAA CAC TAT GGG GCA TCC ACC TGT GAT GGG TGC AAG GGT TTC TTC AGA CGC AGC ATT CGT AAG AGT CAC ATT TAT 49
Ser Cys $\operatorname{Ar}(g)$

TCT TGC AG GTACTTTAAATGCCCTTTTAGGCAAGTTATCTTTACAGATGTTTCAGTTTGGCACGCAAAAAGTAAGAGAAGAAAATTCAGAGTTTTCGTATATCTGTAAGTCTATAAAT ACAATCTCTAAACTGCGGTACAAGTAAGAACGTGTGGGTATGTACACAGGATGTATTCACATAGGTGTTCTTTTTTATGAAACATTTTAGACTTACAGAAGGTATATAAAAAATCCACCTA CCTACCACCCAGCTTAGACCAATATAGTTGAAGCCTTTAATGGACACCTCCTCTGTCACTTTTTCTCCCTCGTCTCCTAGAGGTAACTACCATTGCCATTTCATGTTATCTTTTCCTTGTG TTTCTTTATTATTTTACCACATGTTCATATACCTCTAAACATTTTAAAATCTGCATTTAA: : : : ::: 3 kb: : : : : ::GGGTAAACTTCAGTGGCTGTGGGGTACACTTAAAATTAATA GAAGAATATTTGAAAAGAGAAAAAGCATGAAAATACAGGGCCATGAAGTTCTGACAATAGCCTCCTTCATACTGAAATCATTTAGCAATAAGCAACAAAAACTTGAATGCCAAAGATATTC AAAACTAGGTTTGGCTTTCATGACTCATGATAAAAGTGGTTATGCACAGCAGCCAAAATGAAGATAGTCATTCAGTTTTATTTTACTATAAAGACTAAGTGAGAGGTGAGGCTCGCGTTTT CACGCACATGCTGATTGAGAGACTGGAAAGGTCATAGCTGATTGTTCCTATACCTTCTTTTTATCGAACCAAATTTGACAATACATATTTAAAATCCATTTGTCTGTTTATTTGCTTCTTT Exon $4 \quad 49$

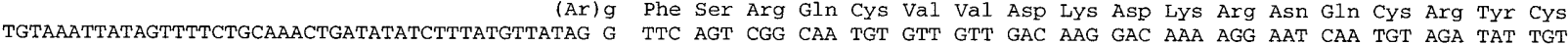
81

Arg Leu Arg Lys Cys Phe Arg Ala Gly Met Lys Lys Glu A(la)

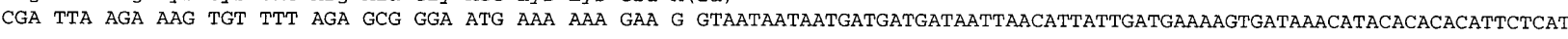
TGATTAGTATAACTTTTACATGAGTTCTAATTTTCCTCAATATAATAAGGAGGAATCTATGATTCCTCAAGTTAAAGAATTTAGCCCAAGGACATCCAGTTATTGAGTGGTATAGCCAGTA TTTATCTGGTGACTACCTGATTATGGATATCATTCTCTCCACCATTACATTGTCCAAGGCAACAAAATTATTGACTAAATGGTATACCAATATTCTGTTAGTGGGTTGAAATTACAGATTT TTTTTTTTTTTTTGAAA : : : : : : $4 \mathrm{~kb}::::::$ :TTTGTGCTCGTGTTTTATAGAGAAAAGAAAATGGAAGTTTCAAAATCTATGACTCATATTTTCTGATAGTGAATACACTCTTAT CTTTGACCTGGTCAATCTTATAGGCAATGCTCTTCCTCTAGTTATCTTATAGTTTTATTAACTTTGAGAGAATAGAAAACCACCCTATTAAAAATACAGCGTGGCGGAACTGGCTGACCCA ACACTTTCTAACACGCATGCTGTCATGAAGTCTCAAACAGCATTGTTTTATGAGCTCTAGTGACAAACCTGACTATTGCTATTTTTGAAAGTAGTGGAGAATGAAAATATCTTTCTTCCAA GGTCTTTTAGAAAGACTTTTTCTCACTTTTTTTACTTAAAAAAAAAACTCCTTAAAATCTATATTCTAACATACACTAAAGAGAAGTGCTCAATAAATACTGTGTTTTTTCCCCCTAG

81 Exon 5

(A) la Val Gln Asn Glu Arg Asp Arg Ile Ser Thr Arg Arg Ser Thr phe Asp Gly Ser Asn Ile Pro Ser Ile Asn Thr Leu Ala Gln Ala CT GTA CAA AAT GAA CGT GAC AGA ATA AGC ACC AGA AGA AGC ACA TTT GAT GGC AGC AAC ATC CCC TCC ATT AAC ACA CTG GCA CAA GCT

Glu Val Arg Ser Arg 116

GAA GTT CGG TCT CGC CAG GTACCTGTGGCACGGCAGCATCAAACCCTATTTAATAAAATCAAATGTCCATGTAGGCATCATGCTAAAATAATCTAAGTTTTTTCAAAGGTGCTCA TTACTAAAATAAGTTACATCTACACACAATTAAGAGTTTTACTTTGAAAGTTTGAGTGGGACTGCTGACTTAATTTTGACTGTTCTTGAACAAGACTAGTTTTAAATGGAAAAATAAAACA AATTTCAGTCTAAGATTGTTTTTTAAAAAGTTTGCTTATAAGTTTGATTAAAAATAATGAATAGCACAAAGCTCTGTAGGCAGTATTTTATTTTATTGATGGAATCTGTACAACCAGCTAC

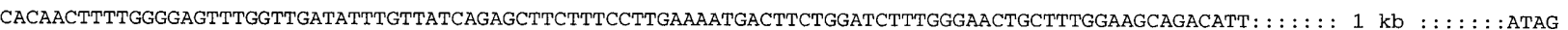


TTCTTGCTTCTTGTCTCCCCAACTGGGTAAACTTTATGAAAACATTTTCTTCATAAACTTCCACAAATTAAAGAAAATATCACCTTTAATTCAAAATAAAATCAAAGAGCATATTACATAT GGATACTATCTATCTATTTTTATAGCCATTPTGAATTGTGAAATTCCTCAAAAGTTGGGGTAAAACTTAGTGTACAGATTTGACCACTAACATAAGATACTGTTCCCAGAGGATGGTGCTA TATATATGAAATGTTTTTAAATTAACTTGTAAACTTATAGGTATTTTATTTTTCAATTTAAATCAGTATTTCATTTTTCAATT'TAAAATGTTTAGTATTTGTCAAGATACTGTAACAACAA Exon $6 \quad 117$ Ile Ser Val TAGTGCCTACTTCTATGGCCTTTACTTATCATAGTCAAAAGAAAAAAGGGGAATGTTGGCCATCTATTATTTTAAATGATAATATAATGCTT'TTCTTAATACTAG ATC TCA GTC Ser Ser Pro Gly Ser Ser Thr Asp Ile Asn Val Lys Lys Ile Ala Ser Ile Gly Asp Val Cys Glu Ser Met Lys Gln Gln Leu Leu Val TCA AGC CCT GGG TCA AGC ACT GAC ATA AAC GTT AAG AAA ATT GCA AGT ATT GGT GAT GTC TGT GAA TCT ATG AAA CAG CAG CTC TTA GTC Leu Val Glu Trp Ala Lys Tyr Ile Pro Ala Phe Cys Glu Leu Pro Leu Asp Asp Gln TTG GTG GAA TGG GCT AAA TAT ATT CCT GCC TTC TGT GAA TTA CCA TTG GAT GAT CAG GTACACATTTAAAACTTTATAAATGCTITAAAAATGCTTCTTGAA CTTTGCTAAGTTTATGTTCTTTCCATATTAT"TTGTAATGCATATGCTATATTACTGTATTTGGCAAAAGATAAATTAGGAGGAAGGATTGGGCTTCCTTACCCATTCACCTTGTTCTATCA

AGAGGCACAACTGTTTGACTTCTATTTTAGGGGTGTAGCAAATTTATTGATTGGACAAGAACTGAACAAAACACTCCTCTGCCCTTTTGGAATTTTAAAAGGGTCATGTGAGGGAGAAAGG TAGATAGCATGTAACTATCATTGTGAGACAAAGTATTAGAAGAGTGAATGTCTGTGAAGCAAATGGTGGATGTTGTGTATTGGCGTTTACTGAAGCTGCTATATAAAACTCCTCAGAAATA CCAGGTCACTCCTAGACAGAAACCACACA : : : : : : $2 \mathrm{~kb}:::::::$ TCTAGAAAGCGGCAGAACCCAGACCTCCCATCTPTTCCTTACAAGAAGTCTTTGCTTTGCTTTTGTTTATTT AGTTTATTACGTGCAGGAGATTATGTTTTATATATGTTTTATCGTTTAGTACTCATGAGTACATGATGAGATTTTTTTTAATCTCTACTCCCATTTTGTAGATGAAAACAAATGAGTAAGT TCAAACGTACTGGTTAACTTACACAAGGTTAAACAGGTAGTAAGTGGCCGTGCTGGGATTTGAACTCTGGTCTGATTCTGTTTCCCTTGCAAACAGTTAACTTGTCTGCTTTTTTTTTTTTT TCTTTCTGCTGCCATTGTATTGATTTGCTAGATAGTTTTAAAATTTTTGAAAAGTAAGTTCTATTGACATGCTATGATAGAAAACTGGAAGGCTTAATGATGGCATGTATCTATGAATACT ATAGTTTGTTAAACAAAAAGTCCCAGGCTTAAAGAACACTGTAAGTTAACAAGACTCATGTCATCTTTTAATCATTATATATTATAATTAATTGTTAAACTGAGAATTTTTCATTAAC 169 Exon 7 Val Ala Leu Leu Arg Ala His Ala Gly Glu His Leu Leu Leu Gly Ala Thr Lys Arg Ser Met Ile Tyr Lys Asp Ile Leu Leu Leu G(ly GTG GCA CTG TTG AGA GCT CAC GCA GGG GAg CAC TTA CTG CTT GGA GCT ACA AAg AGA TCC ATg ATA TAT AAA GAT ATT TTG CTT TTG G GTAA

GTITTTTTTTTAATTTAAGAAGAAATATGCATATTTTATTCTTGCAATATTATACAGGGTCTGTTCTGTATGTACCAAGTATTTACAGACACTGGCAAATGGGCTGTCACTTCAAGGTTT AATTTAATTGGTCCCACTTGGCCTPATTTTTCCTTGCCTTGACTGTATGTAAGTTCAGAGTCCATGAGACAATCAGGGAGCATTAAGATTTAAAAATAAAACAGAGATGTTGCTCAATAAA CATAATATTGAGGTATGTTCAAATTATATCAGAATGAATAATTAGGTAAATTATATGGCTGCCTTATGGAGTCTCAATGTGTTTTCATATTTCCTCAGAGTCTGCTTTTTATTTTACACAC TTTAAAAAAACATGTTTACGTCCTATGATCTTATTGCCCATGATAGCCCACGATATGCTACAAAATGATCAAGTAAAAAAGTATTGGTGGTAATGACTGAACAATTTCGATAGACAGGAAT GCTAGAAGAGGCTAAGGAAGGATAATAGGTGTGGGGTGGTGGGGTAGAGAAGACATAAAGAAAAGACATTTGTCTTCATTAATTGCTCCAATCCCGCTACCTTACTTAACTTAAACAGTTA GGTTTTGAGGGTTGAGATCCTTTTGACACTTACTAGCTCTACACTCTTCTCTGGCATGTGTGAAAATATGACTAGATTATTTTATTTTTGTGCTAGATGTTTTTAGCCCCAAATACAAATAA ATTGAAGACAATATAACAATATTTAATATTATTGCAGGTTTACTATGTAAATGCACTGGTCTAAGTACATTACATGTATATGTATATATATTCTCTCATTTAATCCTTGAAATAGCCCTAA GGGATTAGTACTACTATTTTCAGCCCATTTUGCAGGTGAAGAAATGGAGACAGAGAAAGTTTACCAAAGGTCACAAAGCTAGAAAATAATATAGCCAAGATTCTAAACCACATAATGTGAT TGTGGAGACTATGCCCTGAAATGCTATTAAATACCTCTCTTGAATTTCTAGTAAACATCTAGA : : : : : : 2 kb : : : : : ::TCCTTTACTTAGGTGTGTTAAAACTGTCTCAATAGTGT ATGCCTAGAATGCCTAGAGTATCAAAGTGCTGTTTTTAATCCGCTGTAAACAAGAAAGATTCTTTAGGGTTTGTATTATAGTCATTAGTGAATAAAACGCTAATAGATGTCTGACACAGAG AAAAACTAATCATTATATTTAGATTTTGTCAAATATCAGCATGTTATTAGTCTGCACTTTTGTGTTATCACCAGTGACTGATCCCGTTAAAATGCATGTGATCACATTATTAATGTTCTTG GTCCTTTAAGCATGCTTAAGTTTTTCTAGCATAACTAACAACACCACTGAGAGAGGAAGTACATCACTATTCCTTCAAGGGTAGACTATTTTAAACACCGGTTTGTTAAATTTTAAACAGT Exon 8198 (G) ly Asn Asn Tyr Val Ile His Arg Asn Ser Cys Glu Val Glu GCTGACAATTTGGGGAGACAGGTGGTTATTTTGTTTTGTTTTGTTTTGTTTTCTCTCTCATAG GA AAC AAC TAT GTT ATT CAC CGC AAC AGC TGT GAA GTT GAG Ile Ser Arg Val Ala Asn Arg Val Leu Asp Glu Leu Val Arg Pro Phe Gln Glu Ile Gln Ile Asp Asp Asn Glu Tyr Ala Cys Leu Lys ATT AGC CGT GTG GCC AAT CGT GTT CTA GAT GAG CTG GTT AGA CCA TTT CAA GAA ATC CAG ATT GAT GAC AAT GAG TAT GCT TGT TTA AAG 249

Ala Ile Val Phe Phe Asp Pro A (sp

GCA ATT GTA TTT TTT GAT CCA G GTTGGTTTTCAAAATTCCACTAGAGAATTAATATAATAAAAATTGAACTACTTTMCAATATTAGAATATTTATTGTCTCACACTGTAATTA Exon 9249

(A) sp Ala Lys Gly Leu Ser Asp Pro Val Lys Ile Lys Asn Met Arg Phe Gin Val Gln Ile GGTTAAATCTGTACACTGCCTCTCTTATTCCTTTGTTAG AT GCA AAA GGG CTA AGC GAT CCA GTA AAA ATT AAG AAC ATG AGG TTC CAA GTG CAG ATC

Gly Leu Glu Asp Tyr Ile Asn Asp Arg Gln Tyr Asp Ser Arg Gly Arg Phe Gly Glu Leu Leu Leu Leu Leu Pro Thr Leu Gln Ser Ile GGT TTG GAG GAC TAC ATC AAT GAT CGG CAG TAT GAC TCC CGG GGG AGG TTT GGA GAG TTG CTT CTG CTC CTG CCC ACA CTG CAG AGC ATC

Thr Trp Gln Met Ile Glu Gln Ile Gln Phe Val Lys Leu Phe Gly Met VaI Lys Ile Asp Asn Leu Leu Gln Glu Met Leu Leu Gly G(ly) ACG TGG CAA ATG ATT GAG CAA ATA CAG TTT GTT AAA CTT TTT GGG ATG GTT AAA ATT GAC AAT CTA CTT CAG GAA ATG CTA TTA GGT G GTGA GTACATTGAATAATTTCTACTTTATTGATTAGAATCACACTAAATATAAGTTTGACCTACTGATTTTTTTGTCCATATTTAATTCATCTACTGAAGTTGCTGTGATGCTCCTGAATTTTTT TCCTTCTGCTATGACATAATGAACTCAAGGTTTTACATTTAGAAAATTTGCAAGACCCATCAGAGAGACAAAAATACTTGAACAGATTCAAACCCCCATTCGTTTTTTTTTTTGTTGTTGT TTTGTTTTTTAAGACAGAGTCTCGCTCAGTCGCCAGGCTGGAGCACAGTGGCACAATCTCGGCTCACTGCAACCTCCGACTCCCTAATTCAAGTGATTCTCCTGCTTCAGCCTCCAGAGTA GCTGGGACTACAGGCACACACCACCACGCCTGGCTAATTTTTGTATTTTTAGTAGAGACGGGGTTTCACCGTGTTGATTAGGATGGTCTTGATTTCCTGACCTTGTGATCTGCCAGCCTCG GCCTCCCAAAGTGCTGGGAATACAGGCGTG: : : : : : $: 1 \mathrm{~kb}:::::::$ TTTGCATTCCAATCTAGATTAAATTTCCTTGTAAAATCATTATATTTATTTTTAGTTTTCTGGTGCATAAT TTGTTCCTAATGATATCTTTCTGAAATGAAAGTTGTTCATGCTATTACAGTGATCCCAAATACTATAAACTTGAGGTAAGTGATTCTTGGAATTGCCTTAACAACAGGCAGCGAACCTAAT 
GATTCCAGGAAACGGCACACAAGGTCCATTATTAATACACAGCATAATATGATGCAACATTAATCTAGCCAATGTACAGTGAAAGCTGACATTGTTCTTAATATGCCAGTGGTTCTGTCAA TTTATTTTTGAATTCCAAAAAGCACTTGGCAAAAATAGCAATATTTAAGCTGTCAATCAAAAACATTTGTTTCAAGGTAAACTTGCTGTTCCTGATTAAATATCACTAACACAGCTTCTT Exon $10 \quad 328$

(G) Iy Ala Ser Asn Asp Gly Ser His Leu His His Pro Met His Pro His Leu Ser Gln Asp Pro Leu Thr Gly Gln Thr TTTATCTTTGTAG GG GCT TCC AAT GAT GGC AGT CAT CTC CAT CAT CCA ATG CAT CCA CAT TTG TCT CAA GAC CCA TTA ACT GGA CAA ACT

Ile Leu Leu Gly Pro Met Ser Thr Leu Val His Ala Asp Gln Ile S(er)

ATA CTT TTA GGT CCC ATG TCA ACA CTG GTT CAT GCA GAC CAG ATC T GTAAGTTTATAGACTACTTTTCAACCAAGATATTTCTTAATTACCCAAGAAAAAGTGGAA

TTGTGGGAAAGAATCTATTAGTAACCAGAAATGGCAAAAACTTGGTGTAATTTCCCATCTGAGACTCAGCAGTAGAAGACTGTTATTCAGCTTGTCACTTATTCTCCCTAGACATAAGTTT GCTTCCTAAAATGAAAATGAAAGAAAAATCTTTCTACTTCATAGGTCTGGGGAGCACAAAAGAGTATACAAGTAGTCCTTGCTTTGCATGGTAGTGTGAGACCATAAAAACGACAATGCAA GCTGAAACCATGCAAGGGAATCTTAATAATCAATGGGAAAATTATTTCTTGCTTGGCACCTTCAAAAACGTTTGTCAAAATATTAAGAACTCTCT'TAATGTTTATGGAGACTAAAGAAATA TAATCTTPATACTTATTTTGTAGAATTCAATACAA : : : : : : 4 kb : : : : : : ATATGTCTGAATTAGTGGAATCCAAACCAAATATATTTTTATTTTATGTGAATTTTGATTATAAAA AATATAAACATTGTTTTCTAATATTTGAGAAAACTGTCCTTCTGTTAATTCTTTTCACTGCCTTTTTTTCCTGCTAGGTTTGTTAGAGAATCAAATGCTTATAAATGCTATTAGGAAAGGC AGAAAACACCTTAAAGTAACTATGAAAAAAATTTTTTAACATGACCTCTTTCTATTTAATGTTATTTGGAAAGTAAGGTGAGAGTACTCTCTAACAGTACGTCATGGGCCAATAATGCATT CTACAAATCACTATTCTGTGTAGGATATTTTAAAAACATTTCCAAATTACGCTAATGTGTATTGGTGTTATGTAGGGTTTAATGGGGTGAGGAAGACTGACTGCCACCATTAGACTCAATT Exon $11 \quad 369$

(S)er Thr Pro Glu Thr Pro Leu Pro Ser Pro Pro Gln Gly ser Gly Gln Glu Gln Tyr Lys Ile Ala Ala Asn Gln Ala CTCTGATTCTTTTTCA CA ACT CCT GAA ACC CCA CTC CCT TCC CCA CCA CAA GGC TCT GGG CAA GAA CAG TAC AAA ATA GCT GCA AAC CAA GCA 408

Ser Val Ile Ser His Gln His Leu Ser Lys Gln Lys Gln Leu oP

TCA GTC ATT TCA CAC CAG CAT CTC TCC AAA CAA AAG CAA TTG TGA AAATGTGTTTACTTCAGAACGGCACTACATAAATGTGAAAAGTTGTTGATCTTGAAATATC

TCAAGATAGCACTTTTGGCAAACTCTTAGCCAAGGCTTCTTCATTGGTGCTGTTATAAGATGGTATCCTATTTTCTTGTTTATACGTTCATTCTATTTGTTATTGCTACTATGTGAAACTT G

TCACATGCAACCAATGTATATCTGAgTTTGAagGatgTTTATATAgGGTAGG

Fig. 1. Partial sequence of $H N F 4 G$. The nucleotide and predicted amino acid sequences are shown. The asterisk denotes the 5'-end of the cDNA sequence. This figure is based on the information presented in GenBank accession nos. NM_004133 and AH008234. The approximate size of each intron is indicated. The nucleotide and amino acid polymorphisms found in Japanese subjects are noted. The amino acid sequence shown using the single-letter abbreviations indicates an $\mathrm{N}$-terminal extended form of HNF- $4 \gamma$ that is found in some tissues

was carried out in accordance wtih the principles of the Declaration of Helsinki II. Informed consent was obtained from all subjects before participation in this study.

Screening for mutations in HNF4G. The coding region (exons $3-11)$ and the flanking intron sequences were screened for mutations by amplifying specific regions using the primers listed in Table 1 and then by directly sequencing the PCR products using an ABI PRISM dRhodamine terminator cycle sequencing ready reaction kit (PE Applied Biosystems, Foster City, Calif., USA) and an ABI Prism 377 DNA Sequencer.

Statistical tests. Alleles frequencies were compared using a chisquared test and $p$ less than 0.05 was considered significant.

\section{Results}

The HNF4G is located on chromosome 8 and consists of 12 exons: exons 1 and 2 encode portions of the 5'untranslated region; exon $2 \mathrm{~A}$, an alternative exon found in kidney transcripts and exons 3-11 which contain the protein coding and 3'-untranslated region ([10], GenBank Accession nos. NM_004133 and AH008234). The coding region (exons $3-11$ ) and adjacent introns were screened for mutations in 57 unrelated Japanese subjects with MODY. We found ten sequence variants (Table 1 ), none of which was associated with MODY. They include a silent substitution in exon 6, codon 144 (c.432A/G) and a G-toA substitution in exon 7, codon 190 (c.570G/A) resulting in a conservative Met-to-Ile substitution (M/ I190) in the putative ligand-binding region of HNF$4 \gamma$ protein. Amino acid 190 is also Met in mouse HNF- $4 \gamma$ and the corresponding amino acid in human HNF-4 $\alpha$ is Val. In addition, we observed eight polymorphisms located within introns (Table 1, Fig.1). There was no statistically significant difference in the frequency of any of these polymorphisms between the subjects with MODY and non-diabetic control subjects.

\section{Discussion}

We have found ten polymorphisms in $H N F 4 G$ in Japanese subjects. Direct screening for mutations and association studies suggest that genetic variation in the coding region of $H N F 4 G$ is unlikely to be a major cause of MODY in Japanese patients. Given the heterogeneous nature of MODY and the presence of families with mutations in a related nuclear receptor, 
HNF-4 $\alpha$, there could, however, be rare families with diabetes due to mutations in $H N F 4 G$.

Acknowledgements. This study was supported by the Howard Hughes Medical Institute, the Blum-Kovler Foundation and grants from the United States. Public Health Service (DK20595, DK-44840, DK-48281 and MH/DK-59522), the Japanese Ministry of Health and Welfare (for Research on Human Genome and Gene Therapy), the Japanese Ministry of Science, Culture and Sport (10671084), the Uehara Memorial Foundation and the Naito Memorial Foundation.

\section{References}

1. Yamagata K, Oda N, Kaisaki PJ et al. (1996) Mutations in the hepatocyte nuclear factor- $1 \alpha$ gene in maturity-onset diabetes of the young (MODY3). Nature 384: 455-458

2. Yamagata K, Furuta H, Oda N et al. (1996) Mutations in the hepatocyte nuclear factor- $4 \alpha$ gene in maturity-onset diabetes of the young (MODY1). Nature 384: 458-460

3. Horikawa Y, Iwasaki N, Hara M et al. (1997) Mutation in hepatocyte nuclear factor- $1 \beta$ gene (TCF2) associated with MODY. Nature Genet 17: 384-385

4. Stoffers DA, Ferrer J, Clarke WL, Habener JF (1997) Early-onset type-II diabetes mellitus (MODY4) linked to IPF1. Nature Genet 17: 138-139
5. Shimomura H, Sanke T, Hanabusa Tet al. (1999) Nonsense mutation in the ISL-1 gene (Q310X) found in a type 2 diabetic patient with a strong family history (Abstract). Diabetes 48: [Suppl 1] A405

6. Malecki MT, Jhala US, Antonellis A et al. (1999) Mutations in NEUROD1 are associated with development of type 2 diabetes. Nature Genet 23: 323-328

7. Vionnet N, Stoffel M, Takeda J et al. (1992) Nonsense mutation in the glucokinase gene causes early-onset non-insulin-dependent diabetes mellitus. Nature 356: 721-722

8. Froguel P, Velho G (1999) Molecular genetics of maturityonset diabetes of the young. Trends Endocrinol Metab 10: $142-146$

9. Vaisse C, Kim J, Espinosa R, Le Beau MM, Stoffel M (1997) Pancreatic islet expression studies and polymorphic DNA markers in the genes encoding hepatocyte nuclear factor-3 $\alpha,-3 \beta,-3 \gamma, 4 \gamma$, and -6. Diabetes 46: 1364-1367

10. Plengvidhya N, Antonellis A, Wogan LT et al. (1999) Hepatocyte nuclear factor- $4 \gamma$ : cDNA sequence, gene organization, and mutation screening in early-onset autosomal-dominant type 2 diabetes. Diabetes 48: 2099-2102

11. Hinokio Y, Horikawa Y, Furuta $\mathrm{H}$ et al. (2000) $\beta$-cell transcription factors and diabetes: no evidence for diabetes-associated mutations in the hepatocyte nuclear factor-3 $\beta$ gene $(H N F 3 B)$ in Japanese patients with maturity-onset diabetes of the young. Diabetes 49: 302-305 